\title{
THE EXTENSION OF THE KREIN-ŠMULIAN THEOREM FOR ORDER-CONTINUOUS BANACH LATTICES
}

\author{
ANTONIO S. GRANERO and MARCOS SÁNCHEZ \\ Departamento de Análisis Matemático, Facultad de Matemáticas \\ Universidad Complutense de Madrid, 28040-Madrid, Spain \\ E-mail: AS granero@mat.ucm.es, msaam0003@encina.pntic.mec.es
}

\begin{abstract}
If $X$ is a Banach space and $C \subset X$ a convex subset, for $x^{* *} \in X^{* *}$ and $A \subset X^{* *}$ let $d\left(x^{* *}, C\right)=\inf \left\{\left\|x^{* *}-x\right\|: x \in C\right\}$ be the distance from $x^{* *}$ to $C$ and $\hat{d}(A, C)=\sup \{d(a, C)$ : $a \in A\}$. Among other things, we prove that if $X$ is an order-continuous Banach lattice and $K$ is a w $^{*}$-compact subset of $X^{* *}$ we have: (i) $\hat{d}\left(\overline{\mathrm{co}}^{w^{*}}(K), X\right) \leq 2 \hat{d}(K, X)$ and, if $K \cap X$ is w $^{*}$-dense in $K$, then $\hat{d}\left(\overline{\mathrm{co}}^{w^{*}}(K), X\right)=\hat{d}(K, X)$; (ii) if $X$ fails to have a copy of $\ell_{1}\left(\aleph_{1}\right)$, then $\hat{d}\left(\overline{\mathrm{co}}^{w^{*}}(K), X\right)=\hat{d}(K, X)$; (iii) if $X$ has a 1 -symmetric basis, then $\hat{d}\left(\overline{\mathrm{co}}^{w^{*}}(K), X\right)=\hat{d}(K, X)$.
\end{abstract}

1. Introduction. If $X$ is a Banach space, let $B(X)$ and $S(X)$ be the closed unit ball and unit sphere of $X$, respectively, and $X^{*}$ its topological dual. The weak-topology of $X$ is denoted by w and the weak*-topology of $X^{*}$ by w*. If $C$ is a convex subset of $X^{* *}$, for $x^{* *} \in X^{* *}$ and $A \subset X^{* *}$, let $d\left(x^{* *}, C\right)=\inf \left\{\left\|x^{* *}-x\right\|: x \in C\right\}$ be the distance from $x^{* *}$ to $C$ and $\hat{d}(A, C)=\sup \{d(a, C): a \in A\}$. Observe that: (i) $\hat{d}(\overline{\operatorname{co}}(A), C)=\hat{d}(\operatorname{co}(A), C)=$ $\hat{d}(A, C)$ where $\operatorname{co}(A)$ is the convex hull of $A$; (ii) if $X^{\perp}=\left\{z \in X^{* * *}: z(x)=0, \forall x \in X\right\}$ and $Q: X^{* *} \rightarrow \frac{X^{* *}}{X}$ is the canonical quotient mapping, then:

$$
d\left(x^{* *}, X\right)=\sup \left\{z\left(x^{* *}\right): z \in S\left(X^{\perp}\right)\right\}=\left\|Q x^{* *}\right\| .
$$

With this terminology, the Krein-ŠSmulian Theorem (see [3, p. 51]) states the following: if $X$ is a Banach space and $K \subset X^{* *} \mathrm{a} \mathrm{w}^{*}$-compact subset such that $\hat{d}(K, X)=0$ (thus, $K$ is a weakly compact subset of $X)$, then $\hat{d}\left(\overline{\mathrm{co}} w^{*}(K), X\right)=0$, that is, $\overline{\mathrm{co}} w^{*}(K) \subset X$ and $\overline{\mathrm{co}} w^{*}(K)=\overline{\mathrm{co}}(K)$ is also a weakly compact subset of $X(\overline{\mathrm{co}}(K)=\|\cdot\|$-closure of $\operatorname{co}(K)$ and $\overline{\mathrm{co}}^{w^{*}}(K)=\mathrm{w}^{*}$-closure of $\left.\operatorname{co}(K)\right)$. So, in view of this situation, we can pose two natural questions:

2000 Mathematics Subject Classification: 46B20, 46B26.

Key words and phrases: Krein-Šmulian Theorem, Banach lattices, 1-symmetric spaces.

Supported in part by DGICYT grant MTM2005-00082, grant UCM-910346 and grant UCMBSCH PR27/05-14045

The paper is in final form and no version of it will be published elsewhere. 
(A) If $K \subset X^{* *}$ is a $w^{*}$-compact subset, does the equality $\hat{d}\left(\overline{\operatorname{co}} w^{*}(K), X\right)=\hat{d}(K, X)$ always hold?

The answer to this question is negative. In fact, we constructed (see [5], [7]) a Banach space $X$ such that: (i) there exists a ${ }^{*}$-compact subset $H \subset B\left(X^{* *}\right)$ such that $H \cap X$ is $\mathrm{w}^{*}$-dense in $H, \hat{d}(H, X)=\frac{1}{2}$ and $\hat{d}\left(\overline{\mathrm{co}} w^{*}(H), X\right)=1$; (ii) there exists a $\mathrm{w}^{*}$-compact subset $K \subset B\left(X^{* *}\right)$ such that $\hat{d}(K, X)=\frac{1}{3}$ and $\hat{d}\left(\overline{\mathrm{co}}^{w^{*}}(K), X\right)=1$.

(B) Does there exist a universal constant $1 \leq M<\infty$ such that always $X$ has $M$ control inside its bidual $X^{* *}$, that is, $\hat{d}\left(\overline{\mathrm{co}} w^{*}(K), X\right) \leq M \hat{d}(K, X)$ for every $\mathrm{w}^{*}$-compact subset of $X^{* *}$ ?

The answer to this question is affirmative. In [5] we proved the following result, which extends the Krein-Šmulian Theorem: if $K \subset X^{* *}$ is a $\mathrm{w}^{*}$-compact subset and $Z \subset X$ a subspace of $X$, then $\hat{d}\left(\overline{\mathrm{co}} w^{*}(K), Z\right) \leq 5 \hat{d}(K, Z)$ and, if $Z \cap K$ is $\mathrm{w}^{*}$-dense in $K$, then $\hat{d}\left(\overline{\mathrm{co}} w^{*}(K), Z\right) \leq 2 \hat{d}(K, Z)$. So, in view of these results we have: (i) the universal constant $M$ of our extension of the Krein-Šmulian Theorem satisfies $3 \leq M \leq 5$; (ii) for the category of $\mathrm{w}^{*}$-compact subsets $K \subset X^{* *}$ such that $X \cap K$ is $\mathrm{w}^{*}$-dense in $K$, the constant $M$ is exactly 2 .

Although the answer to question (A) is, in general, negative there are many Banach spaces $X$ for which $\hat{d}\left(\overline{\mathrm{co}} w^{*}(K), X\right)=\hat{d}(K, X)$. This is the case (see [5]), for instance, if $\ell_{1} \nsubseteq X^{*}$, if the unit ball $B\left(X^{*}\right)$ of the dual $X^{*}$ is $w^{*}$-angelic (for example, if $X$ is weakly compactly generated (WCG) or weakly Lindelöf determined (WLD)), if $X=\ell_{1}(I)$, if $K$ is fragmented by the norm of $X^{* *}$, etc.

In order to find classes of Banach space with 1-control in its bidual or, at least, a better control than in the above general case, we examine in this paper the class of Banach lattices. First, we have the following remarks:

(a) In [7, Prop. 10] we have constructed a Banach lattice $X$ and a w*-compact subset $K \subset B\left(X^{* *}\right)$ such that $\hat{d}(K, X)=\frac{1}{3}$ and $\hat{d}\left(\overline{\mathrm{co}} w^{*}(K), X\right)=1$. So, concerning the control inside the bidual, the class of Banach lattices behaves as in the general case.

(b) In [6] we proved that if $\varphi$ is an Orlicz function, $I$ an infinite set and $X=\ell_{\varphi}(I)$ the corresponding Orlicz space, equipped with either the Luxemburg or the Orlicz norm, then for every $\mathrm{w}^{*}$-compact subset $K \subset X^{* *}$ we have $\hat{d}\left(\overline{\mathrm{co}}^{w^{*}}(K), X\right)=\hat{d}(K, X)$ if and only if $\varphi$ satisfies the $\Delta_{2}$ condition at 0 , that is, if and only if $\ell_{\varphi}(I)$ has a 1 -symmetric basis.

In view of these results it is natural to ask the following questions. If $X$ is a Banach space with a 1-symmetric basis, does $X$ have 1-control inside $X^{* *}$ ? What happens if $X$ is an order-continuous Banach lattice? The purpose of this note is to consider these problems. Among other things, we prove that if $X$ is an order-continuous Banach lattice and $K$ is a w*-compact subset of $X^{* *}$ then: (i) $\hat{d}\left(\overline{\mathrm{co}} w^{*}(K), X\right) \leq 2 \hat{d}(K, X)$ and, if $K \cap X$ is $\mathrm{w}^{*}$-dense in $K$, then $\hat{d}\left(\overline{\mathrm{co}}^{w^{*}}(K), X\right)=\hat{d}(K, X)$; (ii) if $X$ fails to have a copy of $\ell_{1}\left(\aleph_{1}\right)$, then $\hat{d}\left(\overline{\mathrm{co}}^{w^{*}}(K), X\right)=\hat{d}(K, X)$; (iii) if $X$ has a 1-symmetric basis, then $\hat{d}\left(\overline{\mathrm{co}} w^{*}(K), X\right)=$ $\hat{d}(K, X)$.

Our notation is standard as in the books [9],[10]. $|A|$ denotes the cardinality of a set $A$, $\omega_{1}$ the first uncountable ordinal and $\aleph_{1}=\left|\omega_{1}\right|$. Let $X$ be a Banach space. If $A$ is a subset 
of $X$, then $[A]$ is the subspace generated by $A$ and $\overline{[A]}$ the closure of $[A]$. The Banach space $X$ is said to be weakly compactly generated (for short, WCG) if there exists a w-compact subset $W$ of $X$ such that $X=\overline{[W]}$. The Banach space $X$ is said to have $M$-control inside its bidual $X^{* *}$ if $\hat{d}\left(\overline{\mathrm{co}}^{w^{*}}(K), X\right) \leq M \hat{d}(K, X)$ for every $\mathrm{w}^{*}$-compact subset $K$ of $X^{* *}$. The notions (countable or uncountable) of 1-unconditional decomposition, 1-unconditional basis, 1-symmetric basis and transfinite basis can be found in [9] and [12]. Concerning the notions of Banach lattices, order-continuity (for short, o-continuity), etc., let us refer the reader to the books [10] and [11].

2. The structure of $X, X^{*}$ and $X^{* *}$ when $X=\sum_{\alpha \in \mathcal{A}} \oplus X_{\alpha}$. Let us consider the structure of $X, X^{*}$ and $X^{* *}$ when $X$ is a 1-unconditional direct sum $X=\sum_{\alpha \in \mathcal{A}} \oplus X_{\alpha}$ of a family of Banach subspaces $\left\{X_{\alpha}: \alpha \in \mathcal{A}\right\}$ of $X$.

Definition 1. A Banach space $X$ is said to be an 1-unconditional direct sum of a family of Banach subspaces $\left\{X_{\alpha}: \alpha \in \mathcal{A}\right\}$ of $X$, for short, $X=\sum_{\alpha \in \mathcal{A}} \oplus X_{\alpha}$ 1-unconditional, when $X=\overline{\left[\cup_{\alpha \in \mathcal{A}} X_{\alpha}\right]}$ and, if $x_{\alpha} \in X_{\alpha}, \epsilon_{\alpha}= \pm 1, \alpha \in \mathcal{A}$, and $A$ is a finite subset of $\mathcal{A}$, then $\left\|\sum_{\alpha \in A} \epsilon_{\alpha} x_{\alpha}\right\| \leq\left\|\sum_{\alpha \in A} x_{\alpha}\right\|$.

If $X=\sum_{\alpha \in \mathcal{A}} \oplus X_{\alpha}$ is a 1-unconditional direct sum, then

(i) For each subset $A \subset \mathcal{A}$ there exists a projection $P_{A}: X \rightarrow X$ such that $\left\|P_{A}\right\|=1$ and $P_{A}(X)=\sum_{\alpha \in A} \oplus X_{\alpha}$.

(ii) Every $x \in X$ has a unique representation of the form $x=\sum_{\alpha \in \mathcal{A}} x_{\alpha}$ with $x_{\alpha} \in$ $X_{\alpha}$ such that the subset $\left\{\alpha \in \mathcal{A}: x_{\alpha} \neq 0\right\}$ is countable, the above series converges unconditionally and $\left\|\sum_{\alpha \in \mathcal{A}} \epsilon_{\alpha} x_{\alpha}\right\|=\|x\|$, where $\epsilon_{\alpha}= \pm 1, \forall \alpha \in \mathcal{A}$.

(iii) If $u \in X^{*}$, the $\alpha$-th coordinate $u_{\alpha}$ of $u$ will be the restriction $u_{\alpha}:=u \uparrow X_{\alpha} \in X_{\alpha}^{*}$ of $u$ to $X_{\alpha}$. We will identify $u$ with the family $\left(u_{\alpha}\right)_{\alpha \in \mathcal{A}}$ of its coordinates.

We consider each dual $X_{\alpha}^{*}$ canonically and isometrically embedded into $X^{*}$ as follows. If $P_{\alpha}: X \rightarrow X_{\alpha}$ is the projection associated to $X_{\alpha}$, then $P_{\alpha}^{*}\left(X_{\alpha}^{*}\right)$ is a subspace of $X^{*}$ isometric to $X_{\alpha}^{*}$. We identify $X_{\alpha}^{*}$ with $P_{\alpha}^{*}\left(X_{\alpha}^{*}\right)$. Consider in $X^{*}$ the closed subspace $Y_{0}:=\overline{\left[\bigcup_{\alpha \in \mathcal{A}} X_{\alpha}^{*}\right]}$, which is actually the 1-unconditional direct sum of the closed subspaces $\left\{X_{\alpha}^{*}: \alpha \in \mathcal{A}\right\}$, that is, $Y_{0}=\sum_{\alpha \in \mathcal{A}} \oplus X_{\alpha}^{*} 1$-unconditional. Let $Y_{0}^{*}$ be the dual of $Y_{0}$.

FACT $1 . Y_{0}^{*}$ can be embedded canonically and isometrically into $X^{* *}$.

Indeed, if $z \in Y_{0}^{*}$, for each $\alpha \in \mathcal{A}$ let $z_{\alpha}:=z \uparrow X_{\alpha}^{*}$ be the $\alpha$-th coordinate of $z$ and identify $z$ with the family $\left(z_{\alpha}\right)_{\alpha \in \mathcal{A}}$ of its coordinates. In order to embed $Y_{0}^{*}$ into $X^{* *}$, define the mapping $h: Y_{0}^{*} \rightarrow X^{* *}$ as follows:

$$
\forall z \in Y_{0}^{*}, \forall u \in X^{*}, h(z)(u)=\sum_{\alpha \in \mathcal{A}} z_{\alpha}\left(u_{\alpha}\right) .
$$

Concerning the definition of $h$ we have to check several things, namely:

(A) First, we need to be sure that the series $\sum_{\alpha \in \mathcal{A}} z_{\alpha}\left(u_{\alpha}\right)$ converges. If $\mathcal{A}_{0} \subset \mathcal{A}$ is a finite subset, then $\sum_{\alpha \in \mathcal{A}_{0}} u_{\alpha} \in Y_{0}$ and, if $\epsilon_{\alpha}= \pm 1, \forall \alpha \in \mathcal{A}$, then

$$
\sum_{\alpha \in \mathcal{A}_{0}} z_{\alpha}\left(\epsilon_{\alpha} u_{\alpha}\right)=z\left(\sum_{\alpha \in \mathcal{A}_{0}} \epsilon_{\alpha} u_{\alpha}\right) \leq\|z\| \cdot\left\|\sum_{\alpha \in \mathcal{A}_{0}} \epsilon_{\alpha} u_{\alpha}\right\| \leq\|z\| \cdot\|u\| .
$$


Thus we get: (i) $\left|\left\{\alpha \in \mathcal{A}: z_{\alpha}\left(u_{\alpha}\right) \neq 0\right\}\right| \leq \aleph_{0}$; (ii) the series $\sum_{\alpha \in \mathcal{A}} z_{\alpha}\left(u_{\alpha}\right)$ converges absolutely and, moreover, $\sum_{\alpha \in \mathcal{A}} z_{\alpha}\left(u_{\alpha}\right) \leq\|z\| \cdot\|u\|$. Therefore $h(z) \in X^{* *}$ with $\|h(z)\| \leq$ $\|z\|, \forall z \in Y_{0}^{*}$, and $h$ is a continuous linear mapping such that $\|h\| \leq 1$.

(B) Let us see that $h$ is an isometry. As $h(z) \uparrow Y_{0}=z, \forall z \in Y_{0}^{*}$, we have

$$
\|h(z)\|=\sup \left\{\langle h(z), u\rangle: u \in B\left(X^{*}\right)\right\} \geq \sup \left\{\langle z, u\rangle: u \in B\left(Y_{0}\right)\right\}=\|z\| .
$$

On the other hand, $\|h(z)\| \leq\|z\|$. So, $h$ is an isometry.

We know that the subspace $Y_{0}^{\perp}:=\left\{z \in X^{* *}:\langle z, y\rangle=0, \forall y \in Y_{0}\right\}$ of $X^{* *}$ is isometrically isomorphic to the dual $\left(\frac{X^{*}}{Y_{0}}\right)^{*}$.

FACT 2. $X^{* *}=h\left(Y_{0}^{*}\right) \stackrel{m}{\oplus} Y_{0}^{\perp}$, that is, $X^{* *}$ is the monotone direct sum of $h\left(Y_{0}^{*}\right)$ and $Y_{0}^{\perp}$. Observe that this means that every $z \in X^{* *}$ has a unique decomposition $z=z_{1}+z_{2}$ with $z_{1} \in h\left(Y_{0}^{*}\right)$ and $z_{2} \in Y_{0}^{\perp}$ so that $\|z\| \geq\left\|z_{1}\right\| \vee\left\|z_{2}\right\|$.

Indeed, it is clear that $h\left(Y_{0}^{*}\right) \cap Y_{0}^{\perp}=\{0\}$. Let $z \in X^{* *}$ and put $w_{1}:=z \uparrow Y_{0}$. Let us see that $z-h\left(w_{1}\right) \in Y_{0}^{\perp}$. For every $\alpha \in \mathcal{A}$ and every $v \in X_{\alpha}^{*}$ we have

$$
\begin{gathered}
\left\langle z-h\left(w_{1}\right), v\right\rangle=\langle z, v\rangle-\left\langle h\left(w_{1}\right), v\right\rangle \\
=\left\langle z\left\lceil X_{\alpha}^{*}, v\right\rangle-\left\langle w_{1 \alpha}, v\right\rangle=\left\langle z\left\lceil X_{\alpha}^{*}, v\right\rangle-\left\langle z \uparrow X_{\alpha}^{*}, v\right\rangle=0 .\right.\right.
\end{gathered}
$$

Thus, $X^{* *}=h\left(Y_{0}^{*}\right) \oplus Y_{0}^{\perp}$. Moreover the above direct sum is monotone because, if $z=$ $z_{1}+z_{2} \in X^{* *}$ with $z_{1} \in h\left(Y_{0}^{*}\right)$ and $z_{2} \in Y_{0}^{\perp}$, we have on the one hand

$$
\|z\| \geq \sup \left\{\left\langle z_{1}+z_{2}, u\right\rangle: u \in B\left(Y_{0}\right)\right\}=\sup \left\{\left\langle z_{1}, u\right\rangle: u \in B\left(Y_{0}\right)\right\}=\left\|z_{1}\right\| .
$$

On the other hand, given $\epsilon>0$, choose $v \in B\left(X^{*}\right)$ such that $\left\|z_{2}\right\|-\frac{\epsilon}{2} \leq\left\langle z_{2}, v\right\rangle$. We know that $\left\langle z_{1}, v\right\rangle=\sum_{\alpha \in \mathcal{A}} z_{1 \alpha}\left(v_{\alpha}\right)$ (where $z_{1 \alpha}:=z \uparrow X_{\alpha}^{*}$ ) so that there exists a finite subset $\mathcal{A}_{0} \subset \mathcal{A}$ such that $\left|\sum_{\alpha \in \mathcal{A} \backslash \mathcal{A}_{0}} z_{1 \alpha}\left(v_{\alpha}\right)\right| \leq \frac{\epsilon}{2}$. Thus, if $u=v-\sum_{\alpha \in \mathcal{A}_{0}} v_{\alpha}$, then $u \in B\left(X^{*}\right)$, $\left\langle z_{2}, u\right\rangle=\left\langle z_{2}, v\right\rangle$ and

$$
\left|\left\langle z_{1}, u\right\rangle\right|=\left|\sum_{\alpha \in \mathcal{A}} z_{1 \alpha}\left(u_{\alpha}\right)\right|=\left|\sum_{\alpha \in \mathcal{A} \backslash \mathcal{A}_{0}} z_{1 \alpha}\left(v_{\alpha}\right)\right| \leq \frac{\epsilon}{2} .
$$

Hence

$$
\left\|z_{2}\right\|-\frac{\epsilon}{2} \leq\left\langle z_{2}, v\right\rangle=\left\langle z_{2}, u\right\rangle \leq\left\langle z_{1}+z_{2}, u\right\rangle+\frac{\epsilon}{2}=\langle z, u\rangle+\frac{\epsilon}{2} \leq\|z\|+\frac{\epsilon}{2} .
$$

As $\epsilon>0$ is arbitrary, we get $\left\|z_{2}\right\| \leq\|z\|$ and so the direct sum $X^{* *}=h\left(Y_{0}^{*}\right) \oplus Y_{0}^{\perp}$ is monotone.

Finally observe that the canonical copy $J(X)$ of $X$ in $X^{* *}$ is inside $h\left(Y_{0}^{*}\right)$ although $J(X) \neq h\left(Y_{0}^{*}\right)$ in general.

3. 1-unconditional direct sums of WCG subspaces. Let us investigate the control inside its bidual of a Banach space which is a 1-unconditional direct sum of WCG subspaces. First, we need the following lemma.

Lemma 2. Let $X$ be a Banach space and $K$ a w-compact subset of $X^{*}$. Given $z \in B\left(X^{* *}\right)$ and $\epsilon>0$, there exists $x \in X$ such that $\|x\| \leq 1+\epsilon$ and

$$
\forall k \in K, z(k)-\epsilon \leq x(k) \leq z(k)+\epsilon .
$$


Proof. Without loss of generality, we suppose that $K$ is convex and symmetric with respect to 0 (otherwise, pick $\overline{\mathrm{co}}(K \cup-K)$ instead of $K$ ). Consider the Banach space $Z=X \oplus_{1} \mathbb{R}$. Then $Z^{*}=X^{*} \oplus_{\infty} \mathbb{R}$ and $Z^{* *}=X^{* *} \oplus_{1} \mathbb{R}$. Let $H_{1}:=\left\{\left(k, z(k)-\frac{\epsilon}{2}\right): k \in K\right\}$ and $H_{2}:=\left\{\left(k, z(k)+\frac{\epsilon}{2}\right): k \in K\right\}$ be two w-compact convex disjoint subsets of $Z^{*}$ such that, if $H=H_{2}-H_{1}$, then $H \subset Z^{*}$ is a w-compact convex subset (and so a $\mathrm{w}^{*}$-compact subset) of $Z^{*}$ fulfilling that $H \cap \stackrel{\circ}{B}\left(0 ; \frac{\epsilon}{2}\right)=\emptyset$. Thus, if we pick $\rho>0$ with $\frac{2}{2+\epsilon} \leq \rho<1$, then $H \cap B\left(0 ; \frac{\rho \epsilon}{2}\right)=\emptyset$. By the Hahn-Banach Theorem there exists a vector $\varphi \in B(Z)$ such that $\langle h, \varphi\rangle \geq \frac{\rho \epsilon}{2}, \forall h \in H$. If $\varphi=x_{0}+t_{0}$, with $x_{0} \in X, t_{0} \in \mathbb{R}$ and $\|\varphi\|=\left\|x_{0}\right\|+\left|t_{0}\right| \leq 1$, then for every $\left(k_{1}, z\left(k_{1}\right)-\frac{\epsilon}{2}\right) \in H_{1}$ and every $\left(k_{2}, z\left(k_{2}\right)+\frac{\epsilon}{2}\right) \in H_{2}$ we have

$$
\varphi\left(\left(k_{2}, z\left(k_{2}\right)+\frac{\epsilon}{2}\right)\right)-\varphi\left(\left(k_{1}, z\left(k_{1}\right)-\frac{\epsilon}{2}\right)\right) \geq \frac{\rho \epsilon}{2} .
$$

Thus

$$
x_{0}\left(k_{2}\right)+t_{0} z\left(k_{2}\right)+t_{0} \frac{\epsilon}{2} \geq x_{0}\left(k_{1}\right)+t_{0} z\left(k_{1}\right)-t_{0} \frac{\epsilon}{2}+\frac{\rho \epsilon}{2},
$$

whence choosing $k_{1}=k_{2}$ in $(3.1)$, we get $t_{0} \epsilon \geq \frac{\rho \epsilon}{2}$, that is, $\frac{\rho}{2} \leq t_{0} \leq 1$. So, $\left\|x_{0}\right\| \leq 1-\frac{\rho}{2}$. Putting $k_{1}=0$ in $(3.1)$ we get

$$
\forall k \in K, x_{0}(k)+t_{0} z(k)+t_{0} \frac{\epsilon}{2} \geq-t_{0} \frac{\epsilon}{2}+\frac{\rho \epsilon}{2} .
$$

Thus

$$
\forall k \in K,-\frac{1}{t_{0}} x_{0}(k) \leq z(k)+\frac{\epsilon}{2} \frac{2 t_{0}-\rho}{t_{0}} \leq z(k)+\epsilon .
$$

On the other hand, putting $k_{2}=0$ in (3.1) we obtain

$$
\forall k \in K, \frac{t_{0}}{2} \epsilon \geq x_{0}(k)+t_{0} z(k)-t_{0} \frac{\epsilon}{2}+\frac{\rho \epsilon}{2} .
$$

Thus

$$
\forall k \in K, z(k)-\epsilon \leq z(k)-\frac{\epsilon}{2} \frac{2 t_{0}-\rho}{t_{0}} \leq-\frac{1}{t_{0}} x_{0}(k) .
$$

Therefore, if $x=-\frac{1}{t_{0}} x_{0}$, then $x$ satisfies the statement of the Lemma.

Proposition 3. Let $X$ be a Banach space, which is a 1-unconditional direct sum of a family $\left\{X_{\alpha}: \alpha \in \mathcal{A}\right\}$ of WCG Banach spaces, we say, $X=\sum_{\alpha \in \mathcal{A}} \oplus X_{\alpha}$. Then

(A) $X$ has 2-control inside the bidual $X^{* *}$.

(B) If the spaces $X_{\alpha}$ are reflexive and $X:=\sum_{\alpha \in \mathcal{A}} \oplus_{\ell_{1}} X_{\alpha}$ (that is, $X$ is the direct $\ell_{1}$-sum of the family $\left\{X_{\alpha}: \alpha \in \mathcal{A}\right\}$ ), then $X$ has 1-control in the bidual $X^{* *}$.

Proof. We adopt the notation of the above paragraphs. So, let $Y_{0}=\sum_{\alpha \in \mathcal{A}} \oplus X_{\alpha}^{*}, X^{* *}=$ $h\left(Y_{0}^{*}\right) \stackrel{m}{\oplus} Y_{0}^{\perp}$, etc. Observe that in the case (B) we have $Y_{0}=\sum_{\alpha \in \mathcal{A}} \oplus_{c_{0}} X_{\alpha}^{*}$, that is, $Y_{0}$ is the direct $c_{0}$-sum of the subspaces $\left\{X_{\alpha}^{*}: \alpha \in \mathcal{A}\right\}$. Let $K_{\alpha}$ be a w-compact subset of $X_{\alpha}$ such that $0 \in K_{\alpha}$ and $X_{\alpha}=\overline{\left[K_{\alpha}\right]}, \alpha \in \mathcal{A}$. In the case (B) we pick $K_{\alpha}:=B\left(X_{\alpha}\right)$. Suppose that there exist a $\mathrm{w}^{*}$-compact subset $K \subset B\left(X^{* *}\right)$ and some real numbers $a, b>0$ such that

(1) $\hat{d}\left(\overline{\mathrm{co}}^{w^{*}}(K), X\right)>b>2 a>2 \hat{d}(K, X)>0$ in the case $(\mathrm{A})$.

(2) $\hat{d}\left(\overline{\mathrm{CO}}^{w^{*}}(K), X\right)>b>a>\hat{d}(K, X)>0$ in the case $(\mathrm{B})$. 
By Lemma 12 of [5] we have

FACT. There exist $\psi \in S\left(X^{* * *}\right) \cap X^{\perp}$ and a $w^{*}$-compact subset $\emptyset \neq H \subset K$ such that for every $w^{*}$-open subset $V$ of $X^{* *}$ with $V \cap H \neq \emptyset$ there exists $\xi \in \overline{\mathrm{co}} w^{*}(V \cap H)$ such that $\langle\psi, \xi\rangle>b$.

Now we proceed step by step:

STEP 1. By the Fact there exists a vector $\xi_{1} \in \overline{\mathrm{co}}^{w^{*}}(H)$ such that $\left\langle\psi, \xi_{1}\right\rangle>b$. Since $B\left(X^{*}\right)$ is $\mathrm{w}^{*}$-dense in $B\left(X^{* * *}\right)$, we can find a vector $x_{1}^{*} \in B\left(X^{*}\right)$ such that $\left\langle\xi_{1}, x_{1}^{*}\right\rangle>b$ and another vector $\eta_{1} \in H$ so that $\left\langle\eta_{1}, x_{1}^{*}\right\rangle>b$. Let $\eta_{1}=v_{1}+w_{1}$ with $v_{1} \in h\left(Y_{0}^{*}\right)$ and $w_{1} \in Y_{0}^{\perp}$. Then $a>d\left(\eta_{1}, X\right) \geq d\left(\eta_{1}, h\left(Y_{0}^{*}\right)\right)=\left\|w_{1}\right\|$, whence

$$
\left\langle v_{1}, x_{1}^{*}\right\rangle=\left\langle\eta_{1}, x_{1}^{*}\right\rangle-\left\langle w_{1}, x_{1}^{*}\right\rangle>b-a .
$$

As $\left\langle v_{1}, x_{1}^{*}\right\rangle=\sum_{\alpha \in \mathcal{A}} v_{1 \alpha}\left(x_{1 \alpha}^{*}\right)>b-a$, we can find a finite subset $\mathcal{A}_{1} \subset \mathcal{A}$ such that, if $y_{1}$ is the restriction of $x_{1}^{*}$ to $\sum_{\alpha \in \mathcal{A}_{1}} \oplus X_{\alpha}$ (so $y_{1}=\sum_{\alpha \in \mathcal{A}_{1}} x_{1 \alpha}^{*} \in B\left(\sum_{\alpha \in \mathcal{A}_{1}} \oplus X_{\alpha}^{*}\right) \subset B\left(Y_{0}\right)$ ), then $\left\langle\eta_{1}, y_{1}\right\rangle=\left\langle v_{1}, y_{1}\right\rangle>b-a$.

SteP 2. Let $V_{1}=\left\{u \in X^{* *}:\left\langle u, y_{1}\right\rangle>b-a\right\}$, which is a $\mathrm{w}^{*}$-open subset of $X^{* *}$ with $V_{1} \cap H \neq \emptyset$, because $\eta_{1} \in V_{1} \cap H$. By the Fact there exists $\xi_{2} \in \overline{\mathrm{co}} w^{*}\left(V_{1} \cap H\right)$ with $\left\langle\psi, \xi_{2}\right\rangle>b$. Let $0<2 \epsilon_{1}<2^{-1} \wedge\left(\left\langle\psi, \xi_{2}\right\rangle-b\right) \wedge\left(a(\hat{d}(K, X))^{-1}-1\right)$. Consider in $X^{* *}$ the subset $L_{1}:=\left\{\xi_{2}\right\} \cup\left(\sum_{\alpha \in \mathcal{A}_{1}} K_{\alpha}\right)$. Clearly $L_{1}$ is a w-compact subset of $X^{* *}$. Moreover, in the case (B), we have $B\left(\sum_{\alpha \in \mathcal{A}_{1}} \oplus_{1} X_{\alpha}\right) \subset L_{1}$. By Lemma 2 there exists a vector $x_{2}^{*} \in X^{*}$ such that $\left\|x_{2}^{*}\right\| \leq 1+\epsilon_{1}$ and

$$
\forall k \in L_{1},\langle\psi, k\rangle-\epsilon_{1}<\left\langle k, x_{2}^{*}\right\rangle<\langle\psi, k\rangle+\epsilon_{1} .
$$

In particular, $\left\langle\xi_{2}, x_{2}^{*}\right\rangle>b+\epsilon_{1}$ and $\left|\left\langle x_{2}^{*}, k\right\rangle\right| \leq \epsilon_{1} \leq 2^{-2}, \forall k \in \sum_{\alpha \in \mathcal{A}_{1}} K_{\alpha}$. Since $\left\langle\xi_{2}, x_{2}^{*}\right\rangle>$ $b+\epsilon_{1}$, we can choose $\eta_{2} \in V_{1} \cap H$ such that $\left\langle\eta_{2}, x_{2}^{*}\right\rangle>b+\epsilon_{1}$ and also $\left\langle\eta_{2}, y_{1}\right\rangle>b-a$ because $\eta_{2} \in V_{1}$. Let $\eta_{2}=v_{2}+w_{2}$ with $v_{2} \in h\left(Y_{0}^{*}\right)$ and $w_{2} \in Y_{0}^{\perp}$. Observe that $\left\|w_{2}\right\|=d\left(\eta_{2}, h\left(Y_{0}^{*}\right)\right) \leq d\left(\eta_{2}, X\right) \leq \hat{d}(K, X)<a$ and $\left|\left\langle w_{2}, x_{2}^{*}\right\rangle\right| \leq\left(1+\epsilon_{1}\right) \hat{d}(K, X) \leq a$. Now we choose $y_{2}$ and $\mathcal{A}_{2}$ in the cases (A) and (B):

CAse A. We have

$$
\left\langle v_{2}, x_{2}^{*}\right\rangle=\left\langle\eta_{2}, x_{2}^{*}\right\rangle-\left\langle w_{2}, x_{2}^{*}\right\rangle \geq\left\langle\eta_{2}, x_{2}^{*}\right\rangle-\left|\left\langle w_{2}, x_{2}^{*}\right\rangle\right|>b-a .
$$

Thus, as $\left\langle v_{2}, x_{2}^{*}\right\rangle=\sum_{\alpha \in \mathcal{A}}\left\langle v_{2 \alpha}, x_{2 \alpha}^{*}\right\rangle>b-a$, we can find a finite subset $\mathcal{A}_{2}$ of $\mathcal{A}$ satisfying $\mathcal{A}_{1} \subset \mathcal{A}_{2} \subset \mathcal{A}$ such that, if $y_{2}$ is the restriction of $x_{2}^{*}$ to $\sum_{\alpha \in \mathcal{A}_{2}} \oplus X_{\alpha}$ (so $y_{2}=\sum_{\alpha \in \mathcal{A}_{2}} x_{2 \alpha}^{*} \in \sum_{\alpha \in \mathcal{A}_{2}} \oplus X_{\alpha}^{*} \subset Y_{0}$ with $\left.\left\|y_{2}\right\| \leq 1+\epsilon_{1}\right)$, then $\left\langle\eta_{2}, y_{2}\right\rangle=\left\langle v_{2}, y_{2}\right\rangle>b-a$. Observe that for every $k \in \bigcup_{\alpha \in \mathcal{A}_{1}} K_{\alpha}$ we have $\psi(k)=0$, whence

$$
\left|\left\langle y_{2}, k\right\rangle\right|=\left|\left\langle x_{2}^{*}, k\right\rangle\right| \leq \epsilon_{1} \leq 2^{-2} .
$$

CASE B. Let $\gamma_{21}:=x_{2}^{*} \uparrow \sum_{\alpha \in \mathcal{A}_{1}} \oplus_{1} X_{\alpha}$ (that is, $\left.\gamma_{21}=\sum_{\alpha \in \mathcal{A}_{1}} x_{2 \alpha}^{*}\right)$ and $\gamma_{22}=x_{2}^{*}-\gamma_{21}$. Since $\left|\left\langle x_{2}^{*}, k\right\rangle\right| \leq \epsilon_{1}, \forall k \in \sum_{\alpha \in \mathcal{A}_{1}} K_{\alpha}$, and $B\left(\sum_{\alpha \in \mathcal{A}_{1}} \oplus_{1} X_{\alpha}\right) \subset \sum_{\alpha \in \mathcal{A}_{1}} K_{\alpha}$, then $\left\|\gamma_{21}\right\| \leq \epsilon_{1}$. So

$$
\left\langle v_{2}, \gamma_{22}\right\rangle=\left\langle\eta_{2}, x_{2}^{*}\right\rangle-\left\langle w_{2}, x_{2}^{*}\right\rangle-\left\langle v_{2}, \gamma_{21}\right\rangle \geq\left\langle\eta_{2}, x_{2}^{*}\right\rangle-\epsilon_{1}-a>b-a .
$$

Since $\left\langle v_{2}, \gamma_{22}\right\rangle=\sum_{\alpha \in \mathcal{A} \backslash \mathcal{A}_{1}}\left\langle v_{2 \alpha}, x_{2 \alpha}^{*}\right\rangle>b-a$, we can find a finite subset $\mathcal{A}_{2} \subset \mathcal{A} \backslash \mathcal{A}_{1}$ such that, if $y_{2}$ is the restriction of $x_{2}^{*}$ to $\sum_{\alpha \in \mathcal{A}_{2}} \oplus X_{\alpha}$ (so $y_{2}=\sum_{\alpha \in \mathcal{A}_{2}} x_{2 \alpha}^{*} \in \sum_{\alpha \in \mathcal{A}_{2}} \oplus X_{\alpha}^{*} \subset Y_{0}$ with $\left.\left\|y_{2}\right\| \leq 1+\epsilon_{1}\right)$, then $\left\langle\eta_{2}, y_{2}\right\rangle=\left\langle v_{2}, y_{2}\right\rangle>b-a$. 
Further we proceed by iteration. We obtain the sequences $\left\{y_{k}: k \geq 1\right\} \subset Y_{0},\left\{\eta_{k}\right.$ : $k \geq 1\} \subset K$ and $\left\{\mathcal{A}_{k}: k \geq 1\right\}, \mathcal{A}_{k} \subset \mathcal{A}$, fulfilling the following conditions:

CASE A. In this case we have:

(i) The finite subsets $\mathcal{A}_{k}$ of $\mathcal{A}$ satisfy $\mathcal{A}_{k} \subset \mathcal{A}_{k+1}$ for $k \geq 1$.

(ii) $y_{k} \in \sum_{\alpha \in \mathcal{A}_{k}} \oplus X_{\alpha}^{*} \subset Y_{0},\left\|y_{k}\right\| \leq 1+\epsilon_{k-1}, k \geq 2$, and $\left\langle\eta_{j}, y_{k}\right\rangle>b-a$ for $j \geq k$ with $j, k \in \mathbb{N}$.

(iii) For every $h \in \bigcup_{\alpha \in \mathcal{A}_{k}} K_{\alpha}$ we have $\left|\left\langle y_{k+1}, h\right\rangle\right| \leq 2^{-k-1}, \forall k \geq 1$.

Let $\mathcal{A}_{0}:=\cup_{n \geq 1} \mathcal{A}_{n}, X_{0}:=\sum_{\alpha \in \mathcal{A}_{0}} \oplus X_{\alpha}$ and let $P_{0}: X \rightarrow X_{0}$ be the canonical projection on $X_{0}$, with norm $\left\|P_{0}\right\|=1$. The space $X$ admits the monotone decomposition

$$
X=X_{0} \stackrel{m}{\oplus} X_{1} \text { where } X_{1}:=\sum_{\alpha \in \mathcal{A} \backslash \mathcal{A}_{0}} X_{\alpha} .
$$

Therefore we get the following monotone decompositions

$$
X^{*}=X_{0}^{*} \stackrel{m}{\oplus} X_{1}^{*}, X^{* *}=X_{0}^{* *} \stackrel{m}{\oplus} X_{1}^{* *}, X^{* * *}=X_{0}^{* * *} \stackrel{m}{\oplus} X_{1}^{* * *}, \text { etc. }
$$

with projections $P_{0}: X \rightarrow X_{0}, P_{0}^{*}: X^{*} \rightarrow X_{0}^{*}, P_{0}^{* *}: X^{* *} \rightarrow X_{0}^{* *}, P_{0}^{* * *}: X^{* * *} \rightarrow X_{0}^{* * *}$, etc. Observe that $P_{0}^{*}\left(y_{k}\right)=y_{k}, \forall k \geq 1$, that is, $y_{k} \in X_{0}^{*}=P_{0}^{*}\left(X^{*}\right), \forall k \geq 1$. Let $\eta_{0}$ be a $\mathrm{w}^{*}$-cluster point of the sequence $\left\{\eta_{k}: k \geq 1\right\}$ in $X^{* *}$. Obviously $\eta_{0} \in K$. Moreover, since $\left\langle\eta_{j}, y_{k}\right\rangle>b-a, \forall j \geq k$, we get $\left\langle\eta_{0}, y_{k}\right\rangle \geq b-a, \forall k \geq 1$. Let $\varphi_{0}$ be a ${ }^{*}$-cluster point of $\left\{y_{k}: k \geq 1\right\}$ in $X^{* * *}$. Then

(i) $\varphi_{0} \in B\left(X^{* * *}\right)$. Actually $\varphi_{0}$ is in $P_{0}^{* * *}\left(X^{* * *}\right)=X_{0}^{* * *}$, that is, $P_{0}^{* * *}\left(\varphi_{0}\right)=\varphi_{0}$.

(ii) By construction $\varphi_{0} K_{\alpha}=0, \forall \alpha \in \mathcal{A}_{0}$. Thus $\varphi_{0} \in X_{0}^{\perp}$, because $\bigcup_{\alpha \in \mathcal{A}_{0}} K_{\alpha}$ generates $X_{0}$.

(iii) $\left\langle\varphi_{0}, \eta_{0}\right\rangle \geq b-a$ because $\left\langle\eta_{0}, y_{k}\right\rangle \geq b-a, \forall k \geq 1$.

Let $W:=P_{0}^{* *}(K) \subset B\left(X_{0}^{* *}\right)$, which is a $\mathrm{w}^{*}$-compact subset of $X_{0}^{* *}$, and $w_{0}=P_{0}^{* *}\left(\eta_{0}\right)$. Obviously $w_{0} \in W$.

Claim 1. $d\left(w_{0}, X_{0}\right)<a$.

Indeed, let $x \in X$ be arbitrary. Then

$$
d\left(w_{0}, X_{0}\right) \leq\left\|w_{0}-P_{0}^{* *} x\right\|=\left\|P_{0}^{* *}\left(\eta_{0}\right)-P_{0}^{* *} x\right\| \leq\left\|\eta_{0}-x\right\| .
$$

That is, $d\left(w_{0}, X_{0}\right) \leq d\left(\eta_{0}, X\right) \leq \hat{d}(K, X)<a$.

Claim 2. $d\left(w_{0}, X_{0}\right) \geq b-a$.

Indeed, as $\varphi_{0} \in B\left(X^{* * *}\right) \cap X_{0}^{\perp}$ and

$$
\left\langle\varphi_{0}, w_{o}\right\rangle=\left\langle\varphi_{0}, P_{0}^{* *} \eta_{0}\right\rangle=\left\langle P_{0}^{* * *} \varphi_{0}, \eta_{0}\right\rangle=\left\langle\varphi_{0}, \eta_{0}\right\rangle \geq b-a,
$$

we conclude that $d\left(w_{0}, X_{0}\right) \geq b-a$.

As $a<b-a$ we get a contradiction which proves the statement in the case (A).

CASE B. In this case we have:

(i) The finite subsets $\mathcal{A}_{k}, k \geq 1$, of $\mathcal{A}$ are disjoint.

(ii) $y_{k} \in \sum_{\alpha \in \mathcal{A}_{k}} \oplus_{0} X_{\alpha}^{*} \subset Y_{0},\left\|y_{k}\right\| \leq 1+\epsilon_{k-1}, k \geq 2$, and $\left\langle\eta_{j}, y_{k}\right\rangle>b-a$ for $j \geq k$ with $j, k \in \mathbb{N}$. 
(iii) For every $n \in \mathbb{N}$ we have $\left\|\sum_{i=1}^{n} y_{i}\right\| \leq 2$.

Let $\eta_{0}$ be a $\mathrm{w}^{*}$-cluster point of the sequence $\left\{\eta_{k}: k \geq 1\right\}$ in $X^{* *}$. Obviously $\eta_{0} \in K$. Moreover, since $\left\langle\eta_{j}, y_{k}\right\rangle>b-a, \forall j \geq k$, we get $\left\langle\eta_{0}, y_{k}\right\rangle \geq b-a>0, \forall k \geq 1$. Thus $\left\langle\eta_{0}, \sum_{i=1}^{n} y_{i}\right\rangle \geq n(b-a), \forall n \geq 1$. Since $\left\|\sum_{i=1}^{n} y_{i}\right\| \leq 2, \forall n \geq 1$, we get a contradiction which proves the statement $(\mathrm{B})$.

Proposition 4. Let $X$ be a Banach space, which is the 1-unconditional direct sum $X=$ $\sum_{\alpha \in \mathcal{A}} \oplus X_{\alpha}$ of the family $\left\{X_{\alpha}: \alpha \in \mathcal{A}\right\}$ of WCG Banach spaces. If $K \subset X^{* *}$ is a $w^{*}$-compact subset such that $K \cap X$ is $w^{*}$-dense in $K$, then $\hat{d}\left(\overline{\mathrm{co}} w^{*}(K), X\right)=\hat{d}(K, X)$.

Proof. The proof is analogous to the one of case (A) of Proposition 3. For reader's convenience we give the details of the proof. Suppose that there exists a $\mathrm{w}^{*}$-compact subset $K \subset B\left(X^{* *}\right)$ such that

$$
\hat{d}\left(\overline{\mathrm{co}}^{w^{*}}(K), X\right)>b>a>\hat{d}(K, X)>0 .
$$

By Lemma 12 of [5] we have:

FACT. There exist $\psi \in S\left(X^{* * *}\right) \cap X^{\perp}$ and a $w^{*}$-compact subset $\emptyset \neq H \subset K$ such that for every $w^{*}$-open subset $V$ of $X^{* *}$ with $V \cap H \neq \emptyset$ there exists $\xi \in \overline{\mathrm{co}} w^{*}(V \cap H)$ with $\langle\psi, \xi\rangle>b$.

STEP 1. By the Fact there exists a vector $\xi_{1} \in \overline{\mathrm{co}}^{w^{*}}(H)$ such that $\left\langle\psi, \xi_{1}\right\rangle>b$. Since $B\left(X^{*}\right)$ is $\mathrm{w}^{*}$-dense in $B\left(X^{* * *}\right)$, we can find a vector $x_{1}^{*} \in B\left(X^{*}\right)$ such that $\left\langle\xi_{1}, x_{1}^{*}\right\rangle>b$. Let $V_{1}=\left\{u \in X^{* *}:\left\langle u, x_{1}^{*}\right\rangle>b\right\}$, which is a $\mathrm{w}^{*}$-open subset of $X^{* *}$ such that $V_{1} \cap H \neq \emptyset$ and so, for the sake of density, also $V_{1} \cap K \cap X \neq \emptyset$. Thus there exists a vector $\eta_{1} \in K \cap X$ such that $\left\langle\eta_{1}, x_{1}^{*}\right\rangle>b$. Since $\eta_{1} \in X$, the support $\mathcal{A}_{1}:=\operatorname{supp}\left(\eta_{1}\right)=\left\{\alpha \in \mathcal{A}: \eta_{1 \alpha} \neq 0\right\}$ of $\eta_{1}$ is countable, we say, $\mathcal{A}_{1}=\left\{\alpha_{1 n}: n \geq 1\right\}$.

SteP 2. As $V_{1} \cap H \neq \emptyset$, by the Fact there exists a vector $\xi_{2} \in \overline{\mathrm{co}} w^{*}\left(V_{1} \cap H\right)$ such that $\left\langle\psi, \xi_{2}\right\rangle>b$. Let $L_{1}:=\bigcup_{i, j=1}^{1} K_{\alpha_{i j}}$ and $\epsilon_{1}:=2^{-1} \wedge\left(\psi\left(\xi_{2}\right)-b\right)$. As $L_{1} \cup\left\{\xi_{2}\right\}$ is a w-compact subset of $X^{* *}$, by Lemma 2 there exists a vector $x_{2}^{*} \in X^{*}$ such that $\left\|x_{2}^{*}\right\| \leq 1+\epsilon_{1}$ and

$$
\forall k \in L_{1} \cup\left\{\xi_{2}\right\},\langle\psi, k\rangle-\epsilon_{1}<\left\langle k, x_{2}^{*}\right\rangle<\langle\psi, k\rangle+\epsilon_{1} .
$$

In particular $\left\langle\xi_{2}, x_{2}^{*}\right\rangle>b$ and $\left|\left\langle x_{2}^{*}, k\right\rangle\right| \leq 2^{-1}, \forall k \in L_{1}$. As $\left\langle\xi_{2}, x_{2}^{*}\right\rangle>b$ and $\xi_{2} \in$ $\overline{\mathrm{co}} w^{*}\left(V_{1} \cap H\right)$, if we put $W_{2}:=\left\{u \in X^{* *} \mid\left\langle u, x_{2}^{*}\right\rangle>b\right\}$, then $W_{2} \cap V_{1} \cap H \neq \emptyset$ and, for the sake of density, also $W_{2} \cap V_{1} \cap K \cap X \neq \emptyset$. Denote $V_{2}:=W_{2} \cap V_{1}$ and choose $\eta_{2} \in V_{2} \cap K \cap X$, which satisfies $x_{i}^{*}\left(\eta_{2}\right)>b, i=1$, 2. Since $\eta_{2} \in X$, the support $\mathcal{A}_{2}:=\operatorname{supp}\left(\eta_{2}\right)=\left\{\alpha \in \mathcal{A}: \eta_{2 \alpha} \neq 0\right\}$ of $\eta_{2}$ is countable, we say, $\mathcal{A}_{2}=\left\{\alpha_{2 n}: n \geq 1\right\}$.

Further we proceed by iteration. We get the sequences $\left\{x_{k}^{*}: k \geq 1\right\} \subset X^{*},\left\{\eta_{k}: k \geq\right.$ $1\} \subset K \cap X,\left\{L_{k}: k \geq 1\right\}$ and $\left\{\mathcal{A}_{k}: k \geq 1\right\}$, such that

(a) $\mathcal{A}_{k}:=\operatorname{supp}\left(\eta_{k}\right)=\left\{\alpha \in \mathcal{A}: \eta_{k \alpha} \neq 0\right\}$ is the support of $\eta_{k}$ and is countable, say, $\mathcal{A}_{k}=\left\{\alpha_{k n}: n \geq 1\right\}, \forall k \geq 1$.

(b) The subsets $L_{k}=\bigcup_{i, j=1}^{k} K_{\alpha_{i j}}$ are w-compact subsets of $B(X), \forall k \geq 1$.

(c) $\left\|x_{k+1}^{*}\right\| \leq 1+\epsilon_{k},\left\langle\eta_{j}, x_{k}^{*}\right\rangle>b, j \geq k \geq 1$, and for every $h \in L_{k}$ we have $\left|\left\langle x_{k+1}^{*}, h\right\rangle\right| \leq 2^{-k}, \forall k \geq 1$. 
Let $\mathcal{A}_{0}:=\bigcup_{n \geq 1} \mathcal{A}_{n}, X_{0}:=\sum_{\alpha \in \mathcal{A}_{0}} \oplus X_{\alpha}$ and let $P_{0}: X \rightarrow X_{0}$ be the canonical projection, with norm $\left\|P_{0}\right\|=1$. Observe that $X_{0}$ is WCG because it is a countable sum of WCG Banach spaces. The space $X$ admits the monotone decomposition

$$
X=X_{0} \stackrel{m}{\oplus} X_{1} \text { where } X_{1}:=\sum_{\alpha \in \mathcal{A} \backslash \mathcal{A}_{0}} X_{\alpha} .
$$

Thus we get the monotone decompositions

$$
X^{*}=X_{0}^{*} \stackrel{m}{\oplus} X_{1}^{*}, X^{* *}=X_{0}^{* *} \stackrel{m}{\oplus} X_{1}^{* *}, X^{* * *}=X_{0}^{* * *} \stackrel{m}{\oplus} X_{1}^{* * *}, \text { etc. }
$$

with projections $P_{0}: X \rightarrow X_{0}, P_{0}^{*}: X^{*} \rightarrow X_{0}^{*}, P_{0}^{* *}: X^{* *} \rightarrow X_{0}^{* *}, P_{0}^{* * *}: X^{* * *} \rightarrow X_{0}^{* * *}$, etc. Observe that $P_{0}\left(\eta_{k}\right)=\eta_{k} \in X_{0}, \forall k \geq 1$. Let $\eta_{0}$ be a $\mathrm{w}^{*}$-cluster point of the sequence $\left\{\eta_{k}: k \geq 1\right\}$ in $X^{* *}$. Obviously $\eta_{0} \in X_{0}^{* *} \cap K$ because $X_{0}^{* *}$ is w*-closed in $X^{* *}$ (actually $X_{0}^{* *}={\overline{X_{0}}}^{w^{*}}$ ) and $\left\{\eta_{k}: k \geq 1\right\} \subset X_{0} \cap K$. Since $\left\langle x_{i}^{*}, \eta_{k}\right\rangle>b, \forall i \leq k$, we get $\left\langle\eta_{0}, x_{i}^{*}\right\rangle \geq b, \forall i \geq 1$, and also $\left\langle\eta_{0}, P_{0}^{*} x_{i}^{*}\right\rangle \geq b, \forall i \geq 1$, because $\left\langle\eta_{0}, P_{0}^{*} x_{i}^{*}\right\rangle=\left\langle P_{0}^{* *} \eta_{0}, x_{i}^{*}\right\rangle=$ $\left\langle\eta_{0}, x_{i}^{*}\right\rangle \geq b$. Let $\varphi_{0}$ be a $\mathrm{w}^{*}$-cluster point of $\left\{P_{0}^{*} x_{k}^{*}: k \geq 1\right\}$ in $X^{* * *}$. Then

(i) $\varphi_{0} \in B\left(X^{* * *}\right)$. Actually $\varphi_{0}$ is in $P_{0}^{* * *}\left(X^{* * *}\right)=X_{0}^{* * *}$, (that is, $\left.P_{0}^{* * *}\left(\varphi_{0}\right)=\varphi_{0}\right)$ because $X_{0}^{* * *}$ is $\mathrm{w}^{*}$-closed in $X^{* * *}$ and $\left\{P_{0}^{*} x_{k}^{*}: k \geq 1\right\} \subset X_{0}^{*} \subset X_{0}^{* * *}$.

(ii) Since for every $k \geq 1$ and every $h \in L_{k}$ we have

$$
\left|\left\langle P_{0}^{*}\left(x_{k+1}^{*}\right), h\right\rangle\right|=\left|\left\langle x_{k+1}^{*}, P_{0}(h)\right\rangle\right|=\left|\left\langle x_{k+1}^{*}, h\right\rangle\right| \leq 2^{-k},
$$

we get $\varphi_{0} \in X_{0}^{\perp}$.

(iii) $\left\langle\varphi_{0}, \eta_{0}\right\rangle \geq b$ because $\left\langle\eta_{0}, P_{0}^{*}\left(x_{k}^{*}\right)\right\rangle \geq b, \forall k \geq 1$.

Let $W:=P_{0}^{* *}(K) \subset B\left(X_{0}^{* *}\right) . W$ is clearly a $\mathrm{w}^{*}$-compact subset of $X_{0}^{* *}$. Let $w_{0}=$ $P_{0}^{* *}\left(\eta_{0}\right)$. Obviously $w_{0} \in W$.

Claim 1. $d\left(w_{0}, X_{0}\right)<a$.

Indeed, first as $W=P_{0}^{* *}(K), X_{0}=P_{0}^{* *}(X)$ and $\left\|P_{0}^{* *}\right\|=1$, we have $\hat{d}\left(W, X_{0}\right) \leq$ $\left\|P_{0}^{* *}\right\| \hat{d}(K, X)<a$. Now it is enough to observe that $w_{0} \in W$.

Claim 2. $d\left(w_{0}, X_{0}\right) \geq b$.

Indeed, since $\varphi_{0} \in B\left(X^{* * *}\right) \cap X_{0}^{\perp}$ and

$$
\left\langle\varphi_{0}, w_{0}\right\rangle=\left\langle\varphi_{0}, P_{0}^{* *} \eta_{0}\right\rangle=\left\langle P_{0}^{* * *} \varphi_{0}, \eta_{0}\right\rangle=\left\langle\varphi_{0}, \eta_{0}\right\rangle \geq b,
$$

we conclude that $d\left(w_{0}, X_{0}\right) \geq b$.

As $a<b$ we get a contradiction which proves the statement.

Let $X$ be a Banach space which admits the decomposition $X=\sum_{\alpha \in \mathcal{A}} \oplus X_{\alpha}$ as a 1-unconditional direct sum of closed subspaces $X_{\alpha}$. We say that the decomposition $X=$ $\sum_{\alpha \in \mathcal{A}} \oplus X_{\alpha}$ is of countable type if for every $u \in X^{*}$ the $\operatorname{support} \operatorname{supp}(u):=\left\{\alpha \in \mathcal{A}: u_{\alpha} \neq\right.$ $0\}$ of $u$ is countable, $\left(u_{\alpha}\right)_{\alpha \in \mathcal{A}}$ being the set of coordinates of $u$, that is, $u_{\alpha}:=u_{\uparrow X_{\alpha}}=u \circ P_{\alpha}$, where $P_{\alpha}: X \rightarrow X_{\alpha}$ is the canonical projection. For instance, if $I$ is an infinite set, $M: \mathbb{R} \rightarrow[0,+\infty]$ an Orlicz function such that its complementary Orlicz function $M^{*}$ (see [2], [9, Chapter 4]) satisfies $M^{*}(t)>0$ for $t>0$, then the Orlicz space $h_{M}(I):=$ $\left\{f \in \mathbb{R}^{I}: \sum_{i \in I} M\left(f_{i} / \lambda\right)<\infty, \forall \lambda>0\right\}$ has countable decomposition (with respect to 
the canonical basis of $\left.h_{M}(I)\right)$, because every element of its dual $h_{M}(I)^{*}:=\ell_{M^{*}}(I)$ has countable support.

Lemma 5. Let $X$ be a Banach space which admits a decomposition $X=\sum_{\alpha \in \mathcal{A}} \oplus X_{\alpha}$ as a 1-unconditional direct sum of closed subspaces. The following statement are equivalent:

(1) The decomposition $X=\sum_{\alpha \in \mathcal{A}} \oplus X_{\alpha}$ is not of countable type.

(2) $X$ has an isomorphic copy of $\ell_{1}\left(\aleph_{1}\right)$ disjointly disposed with respect to the decomposition $X=\sum_{\alpha \in \mathcal{A}} \oplus X_{\alpha}$, that is, there exists a subset $\mathcal{A}_{1} \subset \mathcal{A}$ with cardinality $\left|\mathcal{A}_{1}\right|=\aleph_{1}$ and for each $\alpha \in \mathcal{A}_{1}$ an element $v_{\alpha} \in X_{\alpha}$ so that the family $\left\{v_{\alpha}: \alpha \in \mathcal{A}_{1}\right\}$ is equivalent to the canonical basis of $\ell_{1}\left(\aleph_{1}\right)$.

Proof. (1) $\Rightarrow$ (2). If the decomposition $X=\sum_{\alpha \in \mathcal{A}} \oplus X_{\alpha}$ is not of countable type, there exists some $u \in X^{*}$ such that the subset $\mathcal{A}_{0}:=\left\{\alpha \in \mathcal{A}: u_{\alpha} \neq 0\right\}$ satisfies $\left|\mathcal{A}_{0}\right| \geq \aleph_{1}$, where $u_{\alpha}:=u_{\uparrow X_{\alpha}}=u \circ P_{\alpha}$ and $P_{\alpha}: X \rightarrow X_{\alpha}$ is the canonical projection. By passing to a subset if necessary, we can find a real number $\epsilon>0$, a subset $\mathcal{A}_{1} \subset \mathcal{A}_{0}$ with $\left|\mathcal{A}_{1}\right|=\aleph_{1}$ and a family $\left\{v_{\alpha}: \alpha \in \mathcal{A}_{1}\right\}$ with $v_{\alpha} \in B\left(X_{\alpha}\right)$ so that $\left\langle u, v_{\alpha}\right\rangle=\left\langle u_{\alpha}, v_{\alpha}\right\rangle>\epsilon$. From this we can prove that the family $\left\{v_{\alpha}: \alpha \in \mathcal{A}_{1}\right\}$ is equivalent to the canonical basis of $\ell_{1}\left(\aleph_{1}\right)$ and generates a copy of $\ell_{1}\left(\aleph_{1}\right)$, which is disjointly disposed with respect to the decomposition $X=\sum_{\alpha \in \mathcal{A}} \oplus X_{\alpha}$.

$(2) \Rightarrow(1)$. Let $\mathcal{A}_{1} \subset \mathcal{A}$ be a subset with cardinality $\left|\mathcal{A}_{1}\right|=\aleph_{1}$ and for each $\alpha \in \mathcal{A}_{1}$ let $v_{\alpha}$ be an element of $X_{\alpha}$ so that the family $\left\{v_{\alpha}: \alpha \in \mathcal{A}_{1}\right\}$ is equivalent to the canonical basis $\left\{e_{\alpha}: \alpha \in \mathcal{A}_{1}\right\}$ of $\ell_{1}\left(\mathcal{A}_{1}\right)$. Let $T: \ell_{1}\left(\mathcal{A}_{1}\right) \rightarrow X$ be the isomorphism between $\ell_{1}\left(\mathcal{A}_{1}\right)$ and the closed subspace generated by $\left\{v_{\alpha}: \alpha \in \mathcal{A}_{1}\right\}$ so that $T\left(e_{\alpha}\right)=v_{\alpha}$. Since $T^{*}: X^{*} \rightarrow \ell_{\infty}\left(\mathcal{A}_{1}\right)$ is a quotient mapping and so $T^{*}\left(X^{*}\right)=\ell_{\infty}\left(\mathcal{A}_{1}\right)$, if $w_{0} \in \ell_{\infty}\left(\mathcal{A}_{1}\right)$ is such that $w_{0}(\alpha)=1, \forall \alpha \in \mathcal{A}_{1}$, there exists a vector $u \in X^{*}$ such that $T^{*}(u)=w_{0}$. Then for every $\alpha \in \mathcal{A}_{1}$ we have

$$
\left\langle u, v_{\alpha}\right\rangle=\left\langle u, T e_{\alpha}\right\rangle=\left\langle T^{*} u, e_{\alpha}\right\rangle=\left\langle w_{0}, e_{\alpha}\right\rangle=1,
$$

and this proves that $u$ is an element of $X^{*}$ that does not have countable support with respect to the decomposition $X=\sum_{\alpha \in \mathcal{A}} \oplus X_{\alpha}$.

Proposition 6. Let $X$ be a Banach space that admits a decomposition of countable type $X=\sum_{i \in I} \oplus X_{i}$ as a 1-unconditional direct sum of WCG closed subspaces $\left\{X_{i}: i \in I\right\}$. Then $X$ has 1-control in its bidual $X^{* *}$.

Proof. If $I$ is a countable set, then $X$ is a countable direct sum of WCG Banach subspaces and, so, it is WCG. Thus the statement follows in this case from [5, Cor. 4]. So, suppose in the sequel that $I$ is uncountable. We assume that the statement is not true and we are going to get a contradiction. Let $K$ be a $w^{*}$-compact subset of $X^{* *}$ and $z_{0} \in \overline{\mathrm{co}} w^{w^{*}}(K)$ a vector such that $d\left(z_{0}, X\right)>b>a>\hat{d}(K, X)$. Choose $\psi \in B\left(X^{* * *}\right) \cap X^{\perp}$ such that $\left\langle\psi, z_{0}\right\rangle>b$. We adopt the following notation. For each $i \in I$ let $K_{i} \subset X_{i}$ be a w-compact subset of $X_{i}$ which generates $X_{i}$. If $J$ is a subset of $I$, let $X(J)$ denote the subspace $X(J):=\sum_{i \in J} \oplus X_{i}$ (so $X=X(I)$ ) and let $P_{J}$ denote the canonical projection $P_{J}: X \rightarrow X(J)$ with norm $\left\|P_{J}\right\|=1$. We identify the subspace $P_{J}^{*}\left(X^{*}\right)$ of the dual $X^{*}$ with $X(J)^{*}$. 
STEP 1. Since $\left\langle\psi, z_{0}\right\rangle>b$, there exists $x_{1}^{*} \in B\left(X^{*}\right)$ such that $\left\langle z_{0}, x_{1}^{*}\right\rangle>b$ (because $B\left(X^{*}\right)$ is $\mathrm{w}^{*}$-dense in $\left.B\left(X^{* * *}\right)\right)$. By hypothesis the $\operatorname{support} \operatorname{supp}\left(x_{1}^{*}\right)=\left\{\alpha \in I: 0 \neq x_{1 \alpha}^{*}\right\}:=J_{1}$ of $x_{1}^{*}$ is countable. Let $J_{1}:=\left\{\alpha_{1 j}: j \geq 1\right\}$ and $I_{1}:=J_{1}$. Thus, if $P_{I_{1}}: X \rightarrow \sum_{i \in I_{1}} \oplus X_{i}$ is the corresponding canonical projection, then $P_{I_{1}}^{*}\left(x_{1}^{*}\right)=x_{1}^{*}$ (that is, $\left.x_{1}^{*} \in X\left(I_{1}\right)^{*}\right)$ and moreover

$$
\left\langle P_{I_{1}}^{* *}\left(z_{0}\right), x_{1}^{*}\right\rangle=\left\langle z_{0}, P_{I_{1}}^{*}\left(x_{1}^{*}\right)\right\rangle=\left\langle z_{0}, x_{1}^{*}\right\rangle>b .
$$

STEP 2. Let $K_{\alpha_{11}}$ be the w-compact subset that generates $X_{\alpha_{11}}$, and put $L_{2}:=\left\{z_{0}\right\} \cup$ $K_{\alpha_{11}}$, which is a w-compact subset of $X^{* *}$. Let $\epsilon_{2}=2^{-2} \wedge\left(\left\langle\psi, z_{0}\right\rangle-b\right)$. By Lemma 2 there exists a vector $x_{2}^{*} \in X^{*}$ such that $\left\|x_{2}^{*}\right\| \leq 1+\epsilon_{2}$ and

$$
\forall k \in L_{2},\langle\psi, k\rangle-\epsilon_{2}<\left\langle k, x_{2}^{*}\right\rangle<\langle\psi, k\rangle+\epsilon_{2} .
$$

In particular, $\left\langle z_{0}, x_{2}^{*}\right\rangle>b$ and $\left|\left\langle k, x_{2}^{*}\right\rangle\right| \leq 2^{-2}, \forall k \in K_{\alpha_{11}}$. Let $J_{2}:=\operatorname{supp}\left(x_{2}^{*}\right)$ be the support of $x_{2}^{*}$, which is countable by hypothesis. Put $J_{2}:=\left\{\alpha_{2 j}: j \geq 1\right\}$ and $I_{2}:=J_{1} \cup J_{2}$. Then $P_{I_{2}}^{*}\left(x_{i}^{*}\right)=x_{i}^{*} \in X\left(I_{2}\right)^{*}, i=1,2$, and moreover

$$
\left\langle P_{I_{2}}^{* *}\left(z_{0}\right), x_{i}^{*}\right\rangle=\left\langle z_{0}, P_{I_{2}}^{*}\left(x_{i}^{*}\right)\right\rangle=\left\langle z_{0}, x_{i}^{*}\right\rangle>b, i=1,2 .
$$

STEP 3. Let $L_{3}:=\left\{z_{0}\right\} \cup\left(\bigcup\left\{K_{\alpha_{i j}}: 1 \leq i, j \leq 2\right\}\right)$, which is a w-compact subset of $X^{* *}$. Let $\epsilon_{3}=2^{-3} \wedge\left(\left\langle\psi, z_{0}\right\rangle-b\right)$. By Lemma 2 there exists a vector $x_{3}^{*} \in X^{*}$ such that $\left\|x_{3}^{*}\right\| \leq 1+\epsilon_{3}$ and

$$
\forall k \in L_{3},\langle\psi, k\rangle-\epsilon_{3}<\left\langle k, x_{3}^{*}\right\rangle<\langle\psi, k\rangle+\epsilon_{3} .
$$

In particular, $\left\langle z_{0}, x_{3}^{*}\right\rangle>b$ and $\left|\left\langle k, x_{3}^{*}\right\rangle\right| \leq 2^{-3}, \forall k \in K_{\alpha_{i j}}, 1 \leq i, j \leq 2$. Let $J_{3}:=\operatorname{supp}\left(x_{3}^{*}\right)$ be the support of $x_{3}^{*}$, that is countable by hypothesis, and put $J_{3}:=\left\{\alpha_{3 j}: j \geq 1\right\}$ and $I_{3}:=J_{1} \cup J_{2} \cup J_{3}$. Then $P_{I_{3}}^{*}\left(x_{i}^{*}\right)=x_{i}^{*} \in X\left(I_{3}\right)^{*}, i=1,2,3$, and moreover

$$
\left\langle P_{I_{3}}^{* *}\left(z_{0}\right), x_{i}^{*}\right\rangle=\left\langle z_{0}, P_{I_{3}}^{*}\left(x_{i}^{*}\right)\right\rangle=\left\langle z_{0}, x_{i}^{*}\right\rangle>b, i=1,2,3 .
$$

Further we proceed by iteration. Let $I_{0}:=\bigcup_{n \geq 1} I_{n}$. Observe that $I_{0}$ is a countable subset of $I$ such that $P_{I_{0}}^{*}\left(x_{i}^{*}\right)=x_{i}^{*} \in X\left(I_{0}\right)^{*}, i \geq 1$. Let $\psi_{0} \in B\left(X^{* * *}\right)$ be a w*-cluster point of the sequence $\left\{x_{n}^{*}: n \geq 1\right\}$ in $X^{* * *}$. Then

$(\alpha)$ Since $X\left(I_{0}\right)^{* * *}$ is a $\mathrm{w}^{*}$-closed subset of $X^{* * *}$ and $x_{n}^{*} \in B\left(X\left(I_{0}\right)^{*}\right) \subset B\left(X\left(I_{0}\right)^{* * *}\right)$, $n \geq 1$, we get $\psi_{0} \in B\left(X\left(I_{0}\right)^{* * *}\right)$ and so $P_{I_{0}}^{* * *}\left(\psi_{0}\right)=\psi_{0}$.

( $\beta$ ) Since $\left|\left\langle u, x_{n+1}^{*}\right\rangle\right| \leq 2^{-n-1}, \forall u \in K_{\alpha_{i j}}, 1 \leq i, j \leq n$, and the subset $\bigcup_{i, j \geq 1} K_{\alpha_{i j}}$ generates the space $X\left(I_{0}\right)$, we conclude that $\left.\psi_{0}\right|_{X\left(I_{0}\right)} \equiv 0$, that is, $\psi_{0} \in B\left(X\left(I_{0}\right)^{\mathrm{I}}\right)$.

$(\gamma)$ Since $\left\langle z_{0}, x_{k}^{*}\right\rangle>b, \forall k \geq 1$, then $\left\langle\psi_{0}, z_{0}\right\rangle \geq b$.

Let $H:=P_{I_{0}}^{* *}(K)$ and $w_{0}:=P_{I_{0}}^{* *}\left(z_{0}\right)$. Clearly, $H$ is a $\mathrm{w}^{*}$-compact subset of $X\left(I_{0}\right)^{* *}$ (for the topology $\sigma\left(X\left(I_{0}\right)^{* *}, X\left(I_{0}\right)^{*}\right)$ and $w_{0} \in \overline{\mathrm{co}} w^{*}(H)$.

Claim 1. $\hat{d}\left(H, X\left(I_{0}\right)\right)<a$ and $d\left(w_{0}, X\left(I_{0}\right)\right)<a$.

Indeed

$$
\hat{d}\left(H, X\left(I_{0}\right)\right)=\hat{d}\left(P_{I_{0}}^{* *}(K), P_{I_{0}}^{* *}(X)\right) \leq\left\|P_{I_{0}}^{* *}\right\| \hat{d}(K, X)<a .
$$

By [5, Cor. 4] we have $d\left(w_{0}, X\left(I_{0}\right)\right)<a$, because $X\left(I_{0}\right)$ is a countable direct sum of WCG spaces and so it is WCG.

Claim 2. $d\left(w_{0}, X\left(I_{0}\right)\right) \geq b$. 
Indeed, by $(\gamma)$ we have

$$
\left\langle\psi_{0}, w_{0}\right\rangle=\left\langle\psi_{0}, P_{I_{0}}^{* *}\left(z_{0}\right)\right\rangle=\left\langle P_{I_{0}}^{* * *}\left(\psi_{0}\right), z_{0}\right\rangle=\left\langle\psi_{0}, z_{0}\right\rangle \geq b .
$$

As $\psi_{0} \in X\left(I_{0}\right)^{\perp}$ and $\left\|\psi_{0}\right\| \leq 1$, we get $d\left(w_{0}, X\left(I_{0}\right)\right) \geq b$.

Since $b>a$, we obtain a contradiction which proves the statement.

Proposition 7. Let $X$ be a Banach space that has a transfinite basis $\left\{e_{\alpha}: 1 \leq \alpha<\omega_{1}\right\}$ with constant 1 (that is, if $1 \leq \alpha_{1}<\alpha_{2}<\cdots<\alpha_{n+m}<\omega_{1}$ and $\left(\lambda_{i}\right)_{i=1}^{n+m} \in \mathbb{R}^{n+m}$, then $\left.\left\|\sum_{i=1}^{n} \lambda_{i} e_{\alpha_{i}}\right\| \leq\left\|\sum_{i=1}^{n+m} \lambda_{i} e_{\alpha_{i}}\right\|\right)$ so that every $z \in X^{*}$ has countable support. Then $X$ has 1-control in its bidual $X^{* *}$.

Proof. This proof is analogous to the one of Proposition 6. We suppose that the statement is not true and we are going to obtain a contradiction. Assume that there exist a w*compact subset $K \subset X^{* *}$ and a vector $z_{0} \in \overline{\mathrm{co}}^{w^{*}}(K)$ such that $d\left(z_{0}, X\right)>b>a>$ $\hat{d}(K, X)$. Choose a vector $\psi \in B\left(X^{* * *}\right) \cap X^{\perp}$ such that $\left\langle\psi, z_{0}\right\rangle>b$. We adopt the following notation. If $\beta<\omega_{1}$, let $X(\beta)$ denote the subspace $X(\beta):=\sum_{i<\beta} \oplus\left[e_{i}\right]$ (so $X=X\left(\omega_{1}\right)$ ) and let $P_{\beta}$ be the canonical projection $P_{\beta}: X \rightarrow X(\beta)$ with norm $\left\|P_{\beta}\right\|=1$. We identify the subspace $P_{\beta}^{*}\left(X^{*}\right)$ of $X^{*}$ with $X(\beta)^{*}$.

STEP 1. Since $\left\langle\psi, z_{0}\right\rangle>b$, there exists $x_{1}^{*} \in B\left(X^{*}\right)$ such that $\left\langle z_{0}, x_{1}^{*}\right\rangle>b$ (because $B\left(X^{*}\right)$ is $\mathrm{w}^{*}$-dense in $\left.B\left(X^{* * *}\right)\right)$. By hypothesis the support of $x_{1}^{*}$ is countable and so there exists $\beta_{1}<\omega_{1}$ such that $\operatorname{supp}\left(x_{1}^{*}\right) \subset\left[1, \beta_{1}\right)$. Thus, $P_{\beta_{1}}^{*}\left(x_{1}^{*}\right)=x_{1}^{*}\left(\right.$ that is, $\left.x_{1}^{*} \in X\left(\beta_{1}\right)^{*}\right)$ and moreover

$$
\left\langle P_{\beta_{1}}^{* *}\left(z_{0}\right), x_{1}^{*}\right\rangle=\left\langle z_{0}, P_{\beta_{1}}^{*}\left(x_{1}^{*}\right)\right\rangle=\left\langle z_{0}, x_{1}^{*}\right\rangle>b .
$$

SteP 2. Let $\left[1, \beta_{1}\right)=\left\{\alpha_{1 j}: j \geq 1\right\}$. Since $\left\langle\psi, z_{0}\right\rangle>b$ and $\left\langle\psi, e_{\alpha_{11}}\right\rangle=0$, there exists $x_{2}^{*} \in B\left(X^{*}\right)$ such that $\left\langle z_{0}, x_{2}^{*}\right\rangle>b$ and $\left\langle x_{2}^{*}, e_{\alpha_{11}}\right\rangle=0$. By hypothesis the support of $x_{2}^{*}$ is countable and so there exists $\beta_{1}<\beta_{2}<\omega_{1}$ such that $\operatorname{supp}\left(x_{2}^{*}\right) \subset\left[1, \beta_{2}\right)$. Thus, for $i=1,2$, we have $P_{\beta_{2}}^{*}\left(x_{i}^{*}\right)=x_{i}^{*}$ (that is, $\left.x_{i}^{*} \in X\left(\beta_{2}\right)^{*}\right)$ and moreover

$$
\left\langle P_{\beta_{2}}^{* *}\left(z_{0}\right), x_{i}^{*}\right\rangle=\left\langle z_{0}, P_{\beta_{2}}^{*}\left(x_{i}^{*}\right)\right\rangle=\left\langle z_{0}, x_{i}^{*}\right\rangle>b .
$$

SteP 3. Let $\left[1, \beta_{2}\right)=\left\{\alpha_{2 j}: j \geq 1\right\}$. Since $\left\langle\psi, z_{0}\right\rangle>b$ and $\left\langle\psi, e_{\alpha_{i j}}\right\rangle=0, i, j=1,2$, there exists $x_{3}^{*} \in B\left(X^{*}\right)$ such that $\left\langle z_{0}, x_{3}^{*}\right\rangle>b$ and $\left\langle x_{3}^{*}, e_{\alpha_{i j}}\right\rangle=0, i, j=1,2$. By hypothesis the support of $x_{3}^{*}$ is countable and so there exists $\beta_{2}<\beta_{3}<\omega_{1} \operatorname{such}$ that $\operatorname{supp}\left(x_{3}^{*}\right) \subset\left[1, \beta_{3}\right)$. Thus, for $i=1,2,3$, we have $P_{\beta_{3}}^{*}\left(x_{i}^{*}\right)=x_{i}^{*}$ (that is, $\left.x_{i}^{*} \in X\left(\beta_{3}\right)^{*}\right)$ and moreover

$$
\left\langle P_{\beta_{3}}^{* *}\left(z_{0}\right), x_{i}^{*}\right\rangle=\left\langle z_{0}, P_{\beta_{3}}^{*}\left(x_{i}^{*}\right)\right\rangle=\left\langle z_{0}, x_{i}^{*}\right\rangle>b .
$$

Further we proceed by iteration. Let $\beta_{0}:=\sup \left\{\beta_{n}: n \geq 1\right\}$, that satisfies $\beta_{0}<\omega_{1}$ and $P_{\beta_{0}}^{*}\left(x_{i}^{*}\right)=x_{i}^{*}$, and so $x_{i}^{*} \in X\left(\beta_{0}\right)^{*}, i \geq 1$. Let $\psi_{0} \in B\left(X^{* * *}\right)$ a $\mathrm{w}^{*}$-cluster point of the sequence $\left\{x_{n}^{*}: n \geq 1\right\}$ en $X^{* * *}$. Then

$(\alpha)$ Since $X\left(\beta_{0}\right)^{* * *}$ is a $\mathrm{w}^{*}$-closed subset of $X^{* * *}$ and $x_{n}^{*} \in B\left(X\left(\beta_{0}\right)^{*}\right) \subset B\left(X\left(\beta_{0}\right)^{* * *}\right)$, $n \geq 1$, we get $\psi_{0} \in B\left(X\left(\beta_{0}\right)^{* * *}\right)$ and so $P_{\beta_{0}}^{* * *}\left(\psi_{0}\right)=\psi_{0}$.

$(\beta)$ Since $\left\langle x_{n+1}^{*}, e_{\alpha_{i j}}\right\rangle=0, \forall 1 \leq i, j \leq n$, and the family $\left\{e_{\alpha_{i j}}: 1 \leq i, j\right\}$ generates the space $X\left(\beta_{0}\right)$, we conclude that $\psi_{0} \mid X\left(\beta_{0}\right) \equiv 0$, that is, $\psi_{0} \in B\left(X\left(\beta_{0}\right)^{\perp}\right)$.

$(\gamma)$ Since $\left\langle z_{0}, x_{k}^{*}\right\rangle>b, \forall k \geq 1$, then $\left\langle\psi_{0}, z_{0}\right\rangle \geq b$. 
Let $H:=P_{\beta_{0}}^{* *}(K)$ and $w_{0}:=P_{\beta_{0}}^{* *}\left(z_{0}\right)$. Clearly, $H$ is a $w^{*}$-compact subset of $X\left(\beta_{0}\right)^{* *}$ and $w_{0} \in \overline{\mathrm{CO}}^{w^{*}}(H)$.

Claim 1. $\hat{d}\left(H, X\left(\beta_{0}\right)\right)<a$ and $d\left(w_{0}, X\left(\beta_{0}\right)\right)<a$.

Indeed

$$
\hat{d}\left(H, X\left(\beta_{0}\right)\right)=\hat{d}\left(P_{\beta_{0}}^{* *}(K), P_{\beta_{0}}^{* *}(X)\right) \leq\left\|P_{\beta_{0}}^{* *}\right\| \hat{d}(K, X)<a .
$$

By [5, Cor. 4$]$ we get $d\left(w_{0}, X\left(\beta_{0}\right)\right)<a$, because $X\left(\beta_{0}\right)$ is separable and so WCG.

Claim 2. $d\left(w_{0}, X\left(\beta_{0}\right)\right) \geq b$.

Indeed, by $(\gamma)$ we have

$$
\left\langle\psi_{0}, w_{0}\right\rangle=\left\langle\psi_{0}, P_{\beta_{0}}^{* *}\left(z_{0}\right)\right\rangle=\left\langle P_{\beta_{0}}^{* * *}\left(\psi_{0}\right), z_{0}\right\rangle=\left\langle\psi_{0}, z_{0}\right\rangle \geq b .
$$

As $\psi_{0} \in X\left(\beta_{0}\right)^{\perp}$ and $\left\|\psi_{0}\right\| \leq 1$, we get $d\left(w_{0}, X\left(\beta_{0}\right)\right) \geq b$.

Since $b>a$, we obtain a contradiction, which proves the statement.

Proposition 8. Let $X$ be a Banach space which is the transfinite direct sum of the family $\left\{X_{\alpha}: 1 \leq \alpha<\omega_{1}\right\}$ of WCG subspaces, $X=\sum_{\alpha<\omega_{1}} \oplus X_{\alpha}$, with constant 1 (that is, if $1 \leq \alpha_{1}<\alpha_{2}<\cdots<\alpha_{n+m}<\omega_{1}, x_{\alpha_{i}} \in X_{\alpha_{i}}$ and $\left(\lambda_{i}\right)_{i=1}^{n+m} \in \mathbb{R}^{n+m}$, then $\left.\left\|\sum_{i=1}^{n} \lambda_{i} x_{\alpha_{i}}\right\| \leq\left\|\sum_{i=1}^{n+m} \lambda_{i} x_{\alpha_{i}}\right\|\right)$ so that every $z \in X^{*}$ has countable support. Then $X$ has 1-control in its bidual $X^{* *}$.

Proof. The proof is analogous to the one of Proposition 7 by using the w-compact subsets $K_{\alpha} \subset X_{\alpha}$ that generate $X_{\alpha}, \alpha<\omega_{1}$, and Lemma 2, as in Proposition 6.

4. Application to the order-continuous Banach lattices. First we show that, if $X$ is an o-continuous Banach lattice, then $X$ is a 1-unconditional direct sum of disjoint closed ideals which are WCG. This result is well known (see [1]) but we give its proof for the reader's convenience.

Lemma 9. Let $X$ be an o-continuous Banach lattice with a weak unit $e>0$. Then $X$ is WCG.

Proof. It is well known (see [10, p. 28]) that the interval $[0, e]:=\{x \in X: 0 \leq x \leq e\}$ is a w-compact subset of $X$. Let us see that $X=\overline{[[0, e]]}$, that is, $X$ is the closure of the space generated by $[0, e]$. Pick a positive element $x \in X^{+}$. Then $n e \wedge x \uparrow x$ for $n \rightarrow \infty$, whence $\|x-n e \wedge x\| \downarrow 0$ because $X$ is o-continuous. So $\bigcup_{n \geq 1}[0, n e]=\bigcup_{n \geq 1} n[0, e]$ is dense in the positive cone $X^{+}$. As $X=X^{+}-X^{+}$, we conclude that $X$ is the closure of the subspace generated by $[0, e]$.

Lemma 10. If $X$ is an o-continuous Banach lattice, then $X$ is the 1-unconditional direct sum $X=\sum_{\alpha \in \mathcal{A}} \oplus X_{\alpha}$ of a family of closed ideals $\left\{X_{\alpha}: \alpha \in \mathcal{A}\right\}$ mutually disjoint, such that each $X_{\alpha}$ has weak unit and so it is WCG.

Proof. By [10, 1.a.9] $X$ admits the expression $X=\sum_{\alpha \in \mathcal{A}} \oplus X_{\alpha}$ as a direct sum of a family of closed ideals mutually disjoint $\left\{X_{\alpha}: \alpha \in \mathcal{A}\right\}$ (so as a 1-unconditional direct sum), such that each $X_{\alpha}$ has a weak unit. By Lemma 9 we get the statement. 
Proposition 11. Let $X$ be an o-continuous Banach lattice. If $K$ is a $w^{*}$-compact subset of $X^{* *}$, then $\hat{d}\left(\overline{\mathrm{co}}^{w^{*}}(K), X\right) \leq 2 \hat{d}(K, X)$ and, if $K \cap X$ is $w^{*}$-dense in $K$, then $\hat{d}\left(\overline{\mathrm{co}}^{*}(K), X\right)=\hat{d}(K, X)$.

Proof. This result follows from Lemma 10, Proposition 3 and Proposition 4.

Proposition 12. Let $X$ be an o-continuous Banach lattice that does not have a copy of $\ell_{1}\left(\aleph_{1}\right)$. Then $X$ has 1 -control in its bidual $X^{* *}$.

Proof. Clearly, if $X$ is an o-continuous Banach lattice that does not have a copy of $\ell_{1}\left(\aleph_{1}\right)$, then $X$ admits by Lemma 5 and Lemma 10 a decomposition of countable type $X=\sum_{\alpha \in \mathcal{A}} \oplus X_{\alpha}$ as a 1-unconditional direct sum of closed ideals $X_{\alpha}$ each having weak unit. So, this result follows from Proposition 6.

Proposition 13. Let $X$ be a Banach space with a 1-unconditional basis $\left\{e_{i}: i \in I\right\}$ equivalent to the canonical basis of $\ell_{1}(I)$. Then $X$ has 1-control in its bidual $X^{* *}$.

Proof. The proof is analogous to the one of part (B) of Proposition 3, putting $X_{i}=$ $\left[e_{i}\right], i \in I$, and taking into account that $X^{*}$ and the subspace $Y_{0}$ of $X^{*}$ (see the notation of Proposition 3) are canonically isomorphic to $\ell_{\infty}(I)$ and $c_{0}(I)$, respectively.

A Banach space $X$ has a 1-symmetric basis $\left\{e_{i}: i \in I\right\}$ (see [12, p. 811]) whenever $\left\{e_{i}: i \in I\right\}$ is a 1-unconditional basis of $X$ and, moreover, $\left\{e_{i}: i \in I\right\}$ is symmetric, that is, for any two sequences $\left\{i_{n}: n \geq 1\right\}$ and $\left\{j_{n}: n \geq 1\right\}$ of $I$, the basic sequences $\left\{e_{i_{n}}: n \geq 1\right\}$ and $\left\{e_{j_{n}}: n \geq 1\right\}$ are equivalent. Of course, the canonical bases of non-separable Orlicz spaces, Lorentz spaces, Orlicz-Lorentz spaces, etc., are 1-symmetric bases.

Proposition 14. Let $X$ be a Banach space with a 1-symmetric basis. Then $X$ has 1control in its bidual $X^{* *}$.

Proof. CASE 1. Suppose that every element of the dual $X^{*}$ has countable support. In this case the result follows from Proposition 6.

CAsE 2. Suppose that there exists a vector $u \in B\left(X^{*}\right)$ whit uncountable support. By Proposition 13 it is enough to prove the following claim.

CLAIM. If there exists a vector $u \in B\left(X^{*}\right)$ with uncountable support, then the 1-symmetric basis $\left\{e_{i}: i \in I\right\}$ of $X$ is equivalent to the canonical basis of $\ell_{1}(I)$.

Indeed, $\operatorname{since} \operatorname{supp}(u):=\left\{i \in I: u\left(e_{i}\right) \neq 0\right\}$ is uncountable, we can find a real number $\epsilon>0$ and an uncountable subset $J \subset \operatorname{supp}(u) \operatorname{such}$ that $\left|u\left(e_{i}\right)\right|>\epsilon, \forall i \in J$. Let us prove that the family $\left\{e_{i}: i \in J\right\}$ is equivalent to the basis of $\ell_{1}(J)$. Suppose that the basis $\left\{e_{i}: i \in J\right\}$ is normalized and choose a vector of the form $\sum_{1 \leq k \leq n} \lambda_{k} e_{i_{k}}, i_{k} \in J$. Let $\epsilon_{k}= \pm 1$ so that $u\left(\lambda_{k} \epsilon_{k} e_{i_{k}}\right)=\left|\lambda_{k} u\left(e_{i_{k}}\right)\right| \geq \epsilon\left|\lambda_{k}\right|, 1 \leq k \leq n$. Then

$$
\begin{aligned}
\sum_{1 \leq k \leq n}\left|\lambda_{k}\right| & \geq\left\|\sum_{1 \leq k \leq n} \lambda_{k} e_{i_{k}}\right\|=\left\|\sum_{1 \leq k \leq n} \lambda_{k} \epsilon_{k} e_{i_{k}}\right\| \\
& \geq\left|u\left(\sum_{1 \leq k \leq n} \lambda_{k} \epsilon_{k} e_{i_{k}}\right)\right| \geq \epsilon \sum_{1 \leq k \leq n}\left|\lambda_{k}\right|,
\end{aligned}
$$


and this implies that the family $\left\{e_{i}: i \in J\right\}$ is equivalent to the basis of $\ell_{1}(J)$. As the basis $\left\{e_{i}: i \in I\right\}$ of $X$ is symmetric, finally we conclude that $\left\{e_{i}: i \in I\right\}$ is equivalent to the canonical basis of $\ell_{1}(I)$, and this proves the Claim and completes the proof of the Proposition.

\section{References}

[1] A. V. Bukhvalov, A. I. Veksler and G. J. Lozanovskii, Banach lattices-some Banach aspects of the theory, Russian Math. Surveys 34 (1979), 159-212.

[2] S. Chen, Geometry of Orlicz spaces, Dissert. Math. 356 (1996).

[3] J. Diestel and J. J. Uhl, Vector Measures, Amer. Math. Soc., 1977.

[4] M. Fabian, P. Hájek, V. Montesinos and W. Zizler, A quantitative version of Krein's Theorem, Rev. Mat. Iberoamer. 21 (2005), 237-248.

[5] A. S. Granero, An extension of the Krein- ̌rmulian Theorem, Rev. Mat. Iberoamer. 22 (2006), 93-110.

[6] A. S. Granero, The extension of the Krein-Šmulian Theorem for Orlicz sequence spaces and convex sets, J. Math. Anal. Appl. 326 (2007), 1383-1393.

[7] A. S. Granero, P. Hájek and V. Montesinos, Convexity and $w^{*}$-compactness in Banach spaces, Math. Ann. 328 (2004), 625-631.

[8] M. A. Krasnosel'skiı̌ and Ya. B. Rutickiı̆, Convex Functions and Orlicz Spaces, Noordhoff, Groningen, 1961.

[9] J. Lindenstrauss and L. Tzafriri, Classical Banach Spaces I, Springer-Verlag, Berlin, 1977.

[10] J. Lindenstrauss and L. Tzafriri, Classical Banach Spaces II, Springer-Verlag, Berlin, 1979.

[11] P. Meyer-Nieberg, Banach Lattices, Springer-Verlag, 1992.

[12] I. Singer, Bases in Banach Spaces II, Springer-Verlag, Berlin, 1981. 
